\title{
Mono-energy neutron testing of Single Event Effects
}

\author{
J. Blomgren ${ }^{1, a}$, S. Pomp ${ }^{1}$, J.-C. Bourselier ${ }^{1}$, M. Österlund ${ }^{1}$, A. Prokofiev² ${ }^{2}$ and A. Koning ${ }^{3}$ \\ 1 Uppsala University, Dept of Neutron Research, Box 525, 75120 Uppsala, Sweden \\ 2 Uppsala University, The Svedberg Laboratory, Box 535, 75121 Uppsala, Sweden \\ 3 Nuclear Research and consultancy Group, P.O. Box 25, Petten, The Netherlands
}

\begin{abstract}
The role of mono-energy neutron testing for Single Event Effects (SEE) is outlined. Recent improvements in nuclear reaction theory of relevance to computation of single-event effects from fundamental physics is reported. Older data, as well as recent results obtained with mono-energy neutron testing are well described. Future options of extremely intense mono-energy neutron sources are discussed.
\end{abstract}

\section{Introduction}

Neutron testing of SEEs in memory devices is performed with two major methods. White beam testing, performed at spallation neutron sources, has the advantage of simplicity, in that the facility spectrum resembles the spectrum of atmospheric neutrons, and only a single measurement is needed. A drawback is that these two spectra are not identical. Moreover, different test sites provide different neutron spectra. Thus, corrections have to be estimated if precise results are required, or if data from different white beam facilities should be compared. Unfortunately, accurate corrections are in general very difficult to determine, because the fundamental requirement for such a correction to be determined is knowledge about not only the neutron spectra involved, but also the energy dependence of the SEE sensitivity. The latter cannot even in principle be determined in a white beam, and therefore the corrections have to be estimated from very crude assumptions, resulting in limited precision in the results.

Mono-energy testing has the advantage of being able to overcome these obstacles. By measuring the energy dependence of the SEE sensitivity (i.e., the SEE cross section) at a number of energies, the total SEE sensitivity can be obtained by simply multiplying the SEE sensitivity and the neutron flux versus energy. In principle, this method allows more precise data to be produced than with white beam testing, but with the drawback that measurements have to be performed at several energies (which often is time-consuming). Moreover, the term mono-energy is a truth with qualification. Typically, about half the neutrons are found in a narrow energy interval at maximum neutron energy, while the remaining $50 \%$ constitute a structure-less tail from maximum down to zero energy. The effects of this tail can, however, be corrected for.

A major advantage of using mono-energy testing is that the energy dependence of the SEE sensitivity can provide deep insights into the nuclear reaction mechanisms ultimately causing these effects. This is of great value for the development of computational tools allowing the SEE sensitivity to be estimated already before a new circuit design is taken into production.

\footnotetext{
${ }^{a}$ Presenting author, e-mail: jan.blomgren@tsl.uu.se
}

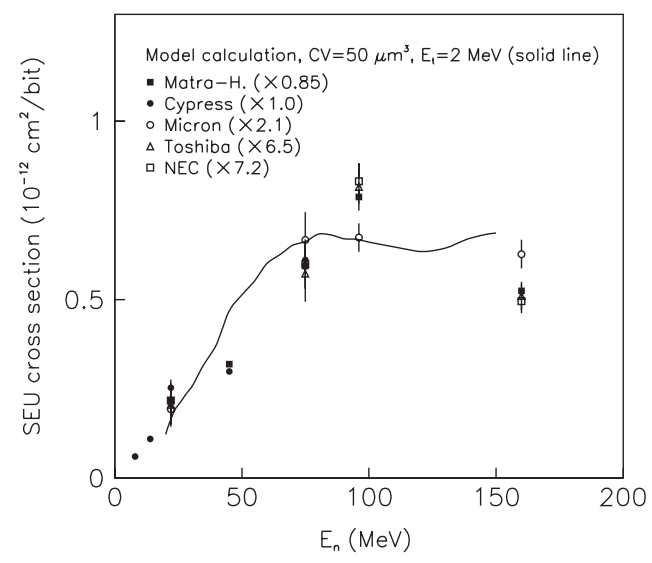

Fig. 1. The SEU cross section for a few memory devices produced in the late 1990s versus neutron energy, reprinted from ref. [5]. The line refers to a model calculation of the SEU cross section using the GNASH code [7] with a similar methodology as TALYS, described elsewhere in the present paper.

The present paper gives a few examples of how previous and recent measurements of the energy dependence of the SEE sensitivity can shed light on the underlying nuclear physics. Recent advances in relevant nuclear theory are described, and the possibilities to develop computational tools for premanufacturing SEE sensitivity estimations are outlined.

\section{Fundamentals on neutron testing}

The ultimate goal of all neutron SEE testing is to establish the sensitivity to the natural neutron flux. In principle, devices could be tested by subjecting them to the natural flux, but this is so time-consuming that it is generally not a realistic option [1]. Naively, one might assume that the ideal testing method would be to have a neutron flux with an energy spectrum identical to the natural flux, but with significantly larger intensity.

In reality, this is difficult to achieve. This is not just a problem related to testing methods, but also to the natural flux itself. The latter is not constant, but depends on a variety 
of natural parameters, like altitude, latitude or weather. In addition, man-made effects play a role. For instance, the presence of shielding material, like buildings or ship hulls, can modify the flux of cosmic particles. Thus, testing in one single neutron field supposed to be identical with the "natural flux" is ultimately impossible. There is no single "natural flux", but many.

If demands on accuracy are modest, white beam testing can be employed. In such facilities, neutrons are created by protons impinging on (most often also stopping in) a target of heavy nuclei, resulting in a strong neutron flux. The energy distribution resembles natural fluxes, but far from perfectly. If normalizing a given artificial and a given natural flux at a single energy to each other, the neutron flux can differ by a factor two in another energy region.

This is also true when comparing different white beam facilities. Different production techniques (i.e., different energy of the incoming beam and different target construction) can result in fairly different energy distributions. Thus, results obtained at two different white beam facilities can be significantly different, up to about a factor two. The standard approach in physics would be to establish correction factors to allow comparisons from, e.g., different facilities, but this is impossible unless information is available on the energy distribution both on the neutron fluxes involved and the energy dependence of the device sensitivity. Since the latter cannot be obtained at white beams, mono-energy testing is required. It should be pointed out that when it comes to correction factors to correlate testing of two different white beam facilities, the correction factors are unique to each component, because the energy dependence of their sensitivity is individual. The sensitivity depends on technical parameters, like critical charge and cell dimensions. Moreover, a correction factor established for a certain device to correlate, e.g., single-bit upset rate, does not apply to other effects in the same device, like multi-bit upsets or latch-ups. This is because the energy dependences of the sensitivities to different types of errors are different.

It can be concluded that if the result should be reliable to about a factor two, white beams can be used, but for better accuracy, mono-energy testing has to be used. Thus, the first added value of mono-energy testing is the potential to reach better accuracy. As will be discussed below, this is not the only reason.

\section{Mono-energy neutron testing}

If accuracy better than what can be obtained at facilities resembling the natural flux (i.e., white beams) is desired, monoenergy testing is the only alternative. Mono-energy testing is presently based on techniques in which a beam of charged particles hit a stationary target. Only a small fraction of the incident beam causes neutron production, and the remaining beam is bent and dumped in a way not to create unmanageable background. The neutrons are primarily produced in the forward direction, but the angular distribution is rather wide, and therefore collimators are required. Due to the fact that neutrons are very penetrating, these collimators have to be thick, of the order of meters, which is one unavoidable limitation of any neutron production technique.
The ideal production reaction should have a large probability (cross section) and as good mono-energy character as possible, i.e., a large fraction of the neutrons should appear in a narrow energy interval. Moreover, the incoming charged particle should preferably be easily accelerated, which in reality means protons. Three suitable reactions are available, protoninduced neutron production on deuterium $\left({ }^{2} \mathrm{H}\right)$ and the two stable lithium isotopes, ${ }^{6} \mathrm{Li}$ and ${ }^{7} \mathrm{Li}$. Deuterium produces a nice spectrum, but requires expensive handling of liquid deuterium. The two lithium isotopes give comparable performance, but ${ }^{6} \mathrm{Li}$ is of strategic importance (it is an important ingredient in thermonuclear weapons) making it difficult to obtain. Thus, ${ }^{7} \mathrm{Li}$ has become the choice at essentially all present monoenergy neutron facilities.

The presence of a low-energy tail is unavoidable with neutron production on a fixed target for neutron energies above about $25 \mathrm{MeV}$, the limit determined by the maximum binding energy difference between the initial and final nuclear systems involved. Since testing has to be performed at higher energies, methods to correct for these tails have to be developed. Such corrections are routinely used in, e.g., nuclear physics research, and a large number of de-convolution codes exist. The most important limiting factors in the final result is a combination of the statistical uncertainty in the raw data, knowledge of the neutron energy spectra, systematic errors in the de-convolution methods (de-convolution is a poorly conditioned mathematical problem, with no single unique solution), and assumptions about the energy dependence of the real cross section.

The latter deserves a special discussion. For a higher nominal neutron energy, a larger fraction of the neutrons are found in the tail. If the real SEE cross section rises with energy all the way to the highest energy point, the correction factors become smaller than if the cross section peaks at a relatively low energy and then decreases. In the latter case, a smaller fraction of the events at the highest nominal energy are due to the full-energy peak, resulting in a larger correction factor, with a correspondingly larger uncertainty.

Thus, the final uncertainty is different for different cases. In general, a final uncertainty of the order of $10 \%$ should be possible to reach with state-of-the-art methods. It is not likely that the final uncertainty can be significantly reduced in a foreseeable future. One ultimately limiting factor is monitoring of the neutron beam flux, which is very difficult to perform to better than $5 \%$ uncertainty in these types of measurements.

\section{Added value of mono-energy testing}

As has been discussed above, the first added value of monoenergy testing is the possibility to suppress the final uncertainty from about a factor two to about $10 \%$. In the discussion of neutron testing, one important aspect often overlooked is the usefulness of large intensity at high energies. White beams can in principle be designed to yield a larger total number of neutrons, but the large majority of the neutrons have low energy. The spectrum typically peaks around one or a few $\mathrm{MeV}$, and falls off approximately as $1 / \mathrm{E}$. This means that the intensity at high energies is much lower than at mono-energy 
facilities. In fact, the low-energy tail of the high-energy fields at intense mono-energy facilities contains more neutrons than the presently most intense white beam. A consequence of this is that testing of effects caused only by high-energy neutrons becomes very time-consuming at white beams. For instance, latch-up effects seems to require at least about $100 \mathrm{MeV}$ in recent devices, and their sensitivity increases rapidly with neutron energy. This means that the testing to reach the same accuracy in the results would require at least an order of magnitude longer irradiation at LAMPF than at TSL $(180 \mathrm{MeV}$ field).

This is also needed when considering other types of effects more complex than standard single-bit SEU in memory devices. Multi-bit upsets (SMU) have been shown to have different energy dependence than single-bit SEU [2], with SMUs becoming more important at high energies. No measurement of energy-resolved SMU cross sections has been published recently, but the general trend of the pioneering paper in 1999 could be well described using fundamental nuclear physics theory, and using the same nuclear theory and modern device parameters results in a similar picture.

Testing is presently often carried out until a preset total number of upsets have been logged. If this is carried out at a white beam, this means that the risk - or chance - that complex errors appear is smaller than in mono-energy testing. Obviously, there is then a risk that the device later shows effects not observed in the testing. Up to now, the discussion has focused on commercial testing. Another advantage of mono-energy beams is their usefulness for research. For instance, monoenergy neutron beam results can in some cases be directly compared with proton beam results (which are inherently of mono-energy character), allowing improved insight into the underlying reaction mechanisms.

\section{Results and discussion}

In the present work, computations with TALYS [3] have been used to calculate energy and angular distributions of all ions created in neutron-induced nuclear reactions on silicon. For reasons described below, not all released reaction products induce upsets. Therefore, a separate post-analysis program has been developed that uses the output from TALYS and converts it to SEE probability, taking only relevant emitted ions into account [4].

In figure 1, the results of the first energy-resolved measurement of neutron-induced single-event upsets (SEU), published in 1998 [5], are presented. Five different memory devices were tested, and it was found that the energy dependence was very similar for all of them, but the absolute sensitivity differed by up to almost an order of magnitude. The oldest components showed lower sensitivity than more recent ones. As can be seen, the sensitivity showed a slow rise from low energy up to about $100 \mathrm{MeV}$, where it saturated or possibly even decreased.

In figure 2, the results of a recent similar test, published in 2005 [6], are shown, however for one device only. It can be seen that the uncorrected result, i.e., raw data before correction for the low-energy neutron tail, partly resembles the results in figure 1 , but with a maximum at a much lower energy and a significant decrease of the SEU cross section at high energies.

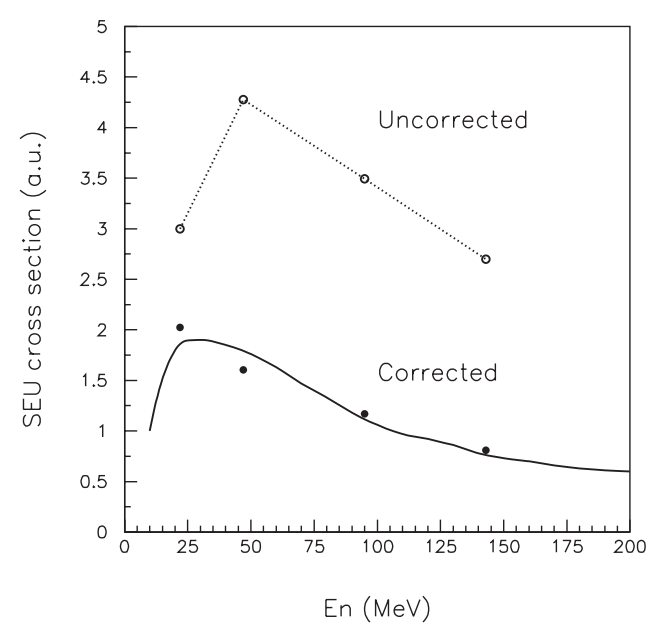

Fig. 2. The SEU cross section for a recent memory devices versus neutron energy, reprinted from ref. [6]. The two data sets are before and after correction for the low-energy tail. The figure is modified by the inclusion of a prediction by the nuclear reaction code TALYS of the cross section. The cross section scale is arbitrary. See the text for details.

The corrected result, however, shows a steady decrease already from the first datum point at $22 \mathrm{MeV}$.

All these results can be understood from fundamental nuclear physics point of view. These effects are caused by neutrons that induce nuclear reactions, releasing charge via emitted ions. (The neutron itself makes no effect, neither do emitted gamma rays.) Whether the released ions cause an upset or not primarily depends on the total charge released, that has to be larger than the critical charge of the component, and on the specific energy loss $(\mathrm{dE} / \mathrm{dx})$, i.e., the amount of energy transferred to the critical volume per length unit of the ion propagation. The latter is important because even if a large total energy in the form of a specific ion is released, but that particular ion does not deposit sufficient energy in a single bit, no upset will result.

All components shown in figure 1 required a rather large critical charge for a bit flip. This had the consequence that relatively exotic nuclear reactions were primarily responsible for the upsets. The cross section for those reactions has a threshold around $10-20 \mathrm{MeV}$ and it increases slowly with energy up to about $50-150 \mathrm{MeV}$ (depending of ion) after which it begins to decrease. Thus, the dependence in figure 1 is in line with expectations from well-known nuclear physics.

Recent components have a much smaller critical charge, but also smaller dimensions of the critical volume. These two effects go in opposite directions when it comes to sensitivity, but it seems as the leading effect is the former, i.e., modern devices require less specific energy loss to cause an SEU. This opens the possibility that other, more common, reaction channels come into play. The dominating neutron-induced nuclear reaction is always elastic scattering. In fact, it is a fundamental quantum mechanics property that elastic scattering must constitute at least half the total neutron cross section. In elastic scattering, the neutron is deflected, leaving the nucleus in its ground state, but with a recoil due to the transferred momentum. This recoiling nucleus has a low energy, resulting 
in a large specific energy loss. Elastic scattering has no energy threshold, so the lowest energy in which it can induce an SEU is primarily determined by the critical charge of the device. As soon as the neutron energy is large enough to cause an upset via the recoils of elastic scattering, this can be expected to be the dominant mechanism, because the cross section is large. In addition, the effect can be expected to peak rather near to the threshold, and then the cross section should slowly decrease with energy.

This is in agreement with the dependence in figure 2 . The solid line shows the prediction by TALYS, presuming reasonable dimensions of the critical volume and charge. The line and the data are normalized to each other, i.e., TALYS describes the trends well, but to get the absolute scale right, also unavailable detailed information on device parameters is needed.

\section{Future facilities}

Until recently, it has been an implicit truth that mono-energy facilities always has a low-energy tail, and that white beams in principle can be made more intense (although at present the leading mono-energy and white facilities actually produce about the same total number of neutrons per second). These presumptions are based on the boundary condition of neutron production on fixed targets. Recently, completely different neutron production techniques have been proposed, in which very intense mono-energy neutron beams can be envisioned.

Two production techniques have been proposed. The first is to use a proton beam of about $1-2 \mathrm{GeV}$ impinging on a combined target and ion source to produce beta-decaying nuclei, which in turn are accelerated and inserted into a storage ring of race-track geometry [8]. Some beta-decaying nuclei emit neutrons immediately after the beta decay ${ }^{1}$. This neutron has a low energy relative to the decaying nucleus. This means that if the nucleus decays in flight, the neutron will be emitted along the direction of motion of the decaying nucleus, with the same velocity. As a consequence, intense mono-energy fluxes will be produced along the straight sections.

It has been estimated that fluxes of $10^{11} \mathrm{n} / \mathrm{s}$ could be achieved, compared with $10^{6}$ today, i.e., a factor 100000 (!) more intense than today. Even if the technique would be a factor 100 less efficient than the design implies, it would still be a factor 1000 more neutrons than presently. Moreover, with such a technique all neutrons would appear in a narrow (few $\mathrm{MeV}$ ) energy range with no low-energy tail. This concept is a spin-off from a conceptual program at the particle physics laboratory CERN, in which similar techniques would be used to produce intense neutrino fluxes for particle physics and cosmology research. The proposed scheme requires infrastructure of the type only CERN can provide, e.g., several coupled high-energy accelerators. Thus, the realization of this technique depends on the realization of the proposed neutrino facility.

A second technique would be to use a similar production as above (1-2 GeV protons on a combined target-ion source) to produce the radioactive nuclide ${ }^{6} \mathrm{He}$, which in turn would be accelerated to hit a target [9]. Roughly, ${ }^{6} \mathrm{He}$ can be described as an alpha particle with two loosely attached neutrons. When hitting a target, the two neutrons are dissociated with a large probability, and continue along the direction of the incident beam with the incident velocity. The charged particles (the remaining ${ }^{6} \mathrm{He}$ and residual ${ }^{4} \mathrm{He}$ ) is bent by a magnet system and a clean neutron beam is produced. This latter technique does not have the potential to produce as intensive fluxes as the beta-decay in flight, but on the other hand it requires much less advanced accelerators. This technique could possibly be installed at existing CERN facilities after some upgrades. Initial estimates indicate a factor a hundred to a thousand larger neutron fluxes than for present facilities to be within reach.

This work was financially supported by the Swedish Nuclear Fuel and Waste Management Company, the Swedish Nuclear Power Inspectorate, Ringhals AB, Barsebäck Power AB, Forsmark Power AB, the Swedish Defense Research Agency, the Swedish Natural Science Research Council, the Swedish Nuclear Safety and Training Centre, and the European Union.

\section{References}

1. J.F. Ziegler, H. Puchner, SER-History, Trends and Challenges (Cypress, 2004).

2. K. Johansson, M. Ohlsson, N. Olsson, J. Blomgren, P.-U. Renberg, IEEE Trans. Nucl. Sci. 46, 1427 (1999).

3. A.J. Koning, S. Hilaire, M.C. Duijvestijn, AIP Conf. Proc. 769, 154 (2005).

4. J.-C. Bourselier, Uppsala University Neutron Physics report 05/07, 2005

5. K. Johansson, P. Dyreklev, B. Granbom, N. Olsson, J. Blomgren, P.-U. Renberg, IEEE Trans. Nucl. Sci. 45, 2519 (1998).

6. M. Olmos, A.V. Prokofiev, R. Gaillard, 2005 IEEE International Reliability Physics Symposium Proceedings, 43rd Annual, San Jose, California, April 17-21, 2005, pp. 696-697.

7. M.B. Chadwick, H.H. Barschall, R.S. Caswell, P.M. DeLuca Jr., G.M. Hale, D.T.L. Jones, R.E. MacFarlane, J.P. Meulders, H. Schuhmacher, U.J. Schrewe, A. Wambersie, P.G. Young, Med. Phys. 26, 974 (1999) ; and ICRU Report 63 (International Commission on Radiation Units and Measurements, Bethseda, MD, 2000).

8. CERN beta beam project, http://beta-beam.web.cern.ch/betabeam/.

9. I. Tanihata, M. Lindroos (private communication).

\footnotetext{
1 This effect is of major importance for the stability of nuclear power reactors.
} 